\title{
Ferromagnetism in bare gold nanoagglomerates produced by nanocluster deposition
}

\section{Venäläinen, Annika}

2018-05-15

Venäläinen , A , Jalkanen , P , Tuboltsev , V , Meinander , N K, Savin , A \& Räisänen , J 2018 , ' Ferromagnetism in bare gold nanoagglomerates produced by nanocluster deposition ' , Journal of Magnetism and Magnetic Materials , vol. 454 , pp. 57-60 . https://doi.org/10.1016/j.jmmm.2018.01.041

http://hdl.handle.net/10138/312427

https://doi.org/10.1016/j.jmmm.2018.01.041

cc_by_nc_nd

acceptedVersion

Downloaded from Helda, University of Helsinki institutional repository.

This is an electronic reprint of the original article.

This reprint may differ from the original in pagination and typographic detail.

Please cite the original version. 


\title{
Ferromagnetism in bare gold nanoagglomerates produced by nanocluster deposition
}

\author{
Annika Venalainen, ${ }^{1}$ a) Pasi Jalkanen, ${ }^{1}$ Vladimir Tuboltsev,${ }^{1}$ Kristoffer Meinander, ${ }^{1}$ Alexandre Savin, ${ }^{2}$ and Jyrki \\ Raisanen ${ }^{1}$ \\ 1) Division of Materials Physics, Department of Physics, University of Helsinki, P.O.Box 43, \\ FIN-00014 University of Helsinki, Finland \\ ${ }^{2)}$ Low Temperature Laboratory, Department of Applied Physics, Aalto University School of Science, P.O. Box 15100, \\ FI-00076 Aalto, Finland
}

(Dated: 7 August 2017)

Recent research has shown unconventional magnetic properties in nanosized gold systems. These effects have mostly been detected in functionalized gold nanoparticles as well as in gold nanocrystalline films. We demonstrate ferro- and superparamagnetic behaviour in assemblies of bare gold nanoclusters. This is demonstrated by the characteristic ferromagnetic hysteresis with the temperature dependent saturation magnetization, remanence and coercivity in aggregates of small clusters. The detected magnetization, is caused by the interaction between the separate clusters exhibiting an core-shell structure, and dependent on the total amount of gold confined in the samples. The behaviour is analogous to that of transition metal clusters.

Keywords: Au cluster deposition, nanoagglomerate, ferromagnetism, superparamagnetism

\section{INTRODUCTION}

Bulk gold is well known to be diamagnetic. Recently however there have been both experimental and theoretical work indicating that departure from diamagnetism develops, while going down to nanoscale gold systems. These effects have mostly been exhibited by gold nanocrystalline films as well as functionalized gold nanoparticles ${ }^{1}$. Only a few papers have been published on the ferromagnetic behaviour of separate bare gold nanoclusters, these do not however treat the total volume dependence of magnetic behaviour in gold ${ }^{2-5}$.

It is believed that the observed magnetic behaviour that departures from the bulk diamagnetism is an intrinsic property of nanosized gold with large surfaceto-volume ratio ${ }^{6-9}$. Computer simulations also predict size-dependent spin polarization and ferromagnetism in bare gold nanoparticles and thin films due to the effect of surfaces at the nanoscale and strong spin-orbit coupling in gold ${ }^{10-13}$. Different available theoretical models that predict and explain the positive magnetization and ferromagnetism in bare gold nanoparticles as well as experimental results differ in detail. Overall they still have a common denominator, which is the existence of a core-shell magnetic structure with a bulk-like diamagnetic core and a shell of differently ordered surface magnetic moments ${ }^{10-15}$.

Sato et al. have investigated the temperature dependence of Au nanocluster magnetization as well as the impact of exposure to air on their magnetization ${ }^{2,3}$. Li et al. have investigated the intrinsic magnetic moment of $4 \mathrm{~nm}$ bare face-centered cubic (fcc) Au nanoparticles ${ }^{4,5}$. Previously the size- and temperature dependent structural transitions in gold nanoparticles have been studied ${ }^{16}$,

\footnotetext{
a)Electronic mail: annika.venalainen@helsinki.fi
}

but not in the connection with their magnetic properties. Further experimental studies into the behaviour of similar systems is desirable.

Previously we have reported on the ferromagnetism in nanocrystalline gold thin films ${ }^{17}$. In this work we contribute to the controversial subject of magnetism in gold nanostructures, by presenting experimental evidence of ferro- and superparamagnetic behaviour in assemblies of $\mathrm{Au}$ nanoclusters and complement the available data existing for thin films and individual gold clusters. It is shown that the magnetic behaviour depends on the total amount of gold confined in the samples.

Magnetic phenomena are very sensitive to the atomic environment. As the cluster interactions change due to changes in interaction range and degree, the magnetic behaviour is also influenced. When clusters are separate from each other (below the percolation threshold) long range dipolar forces and short range exchange forces act. As isolated particles evolve by agglomeration, exchangecoupled agglomerates will be produced. The agglomerates interact with each other via dipolar forces. When the percolation threshold is exceeded the behaviour of cluster assemblies is dominated by inter-cluster exchange coupling $^{18}$. According to Löffler et.al. the exchange interaction at the boundaries between particles is weaker than the intra-particle atomic exchange which further reinforces the image of separate but interacting clusters ${ }^{19}$. Chudnovsky et al. ${ }^{20-22}$ have studied the magnetic behaviour of nanostructured ferromagnetic materials. They have described how an increase in the inter-particle exchange of the cluster configurations evolves to a correlated super-spin glass (CSSG) in which the magnetization vectors of neighbouring particles are nearly aligned. The disordered CSSG state is fragile and application of a small field will produce a 'ferromagnet with wandering axes' (FWA). If gold clusters are ferromagnetic they should also produce similar effects as they start to interact at close proximity and overcome the percolation 
threshold. Therefore, our goal in this work was to observe experimentally how magnetization of gold nanoagglomerates depends on their aggregation state that allows to conclude on ferromagnetism in bare gold nanoclusters.

\section{MATERIALS AND METHODS}

In the present work bare gold nanoparticles were produced with a condensation-cell-type cluster aggregation source (NC200, Oxford Applied Research), charged with a gold target of $99.999 \%$ purity. The gold clusters were deposited at room temperature directly on a template, a lightweight homogeneous plastic straw provided by Quantum Design as a sample holder for ultrahighsensitivity measurements. The magnetic properties were evaluated using an ultra-high-sensitive magnetometry based on a superconducting quantum interference device, SQUID (Quantum Design MPMS-XL7), providing a magnetic field up to $70 \mathrm{kOe}$ at temperatures from 1.8 to $400 \mathrm{~K}$.

Before the actual SQUID measurements of the nanocluster deposited gold, the background signal caused by the templates shape, local deformations, material defects, and non-homogeneity was measured. This was done by measuring the template (with no gold deposited) with all of the experimental conditions the same as during the actual measurements. The resulting data was then subtracted from the actual magnetization data measured from gold deposited in the templates. This was done in order to verify that the presented results originate only from the deposited gold.

Special care was taken to prevent the deposited gold as well as the template to be exposed to parasitic magnetic impurities. To ensure that the effect from native impurities in the substrate and deposited gold was negligible, the impurity level was evaluated by inductively coupled plasma (ICP) mass spectrometry and elastic recoil detection analysis (ERDA), after the magnetization measurements. With all the precautions taken the total concentration of all trace elements in the gold as well as the template were at a level of $10 \mathrm{ppm}$, and thereby these impurities do not influence our results ${ }^{17}$.

The morphology of the samples was examined by highresolution transmission electron microscopy, HR-TEM (JEOL JEM-2200FS) and atomic force microscopy, AFM (Veeco AutoProbe CP-Research). For the HR-TEM measurements the gold clusters were deposited on carbon holey films $\sim 12 \mathrm{~nm}$ thick (Quantifoil Micro Tools $\mathrm{GmbH}$ ). We assume that the morphology of the deposited gold was not affected by the templates, as both the Quantifoil carbon film and the material for the plastic straws used in the magnetization measurements are chemically non-reactive.

\section{RESULTS AND DISCUSSION}

The energy of deposited Au clusters in our case was $\sim 0.3 \mathrm{eV}$ per atom ${ }^{17,23,24}$. This leads to the formation of well developed branches and porous like morphology of the resulting sample. The clusters act as building blocks, softly landing on top of each other and undergoing minor agglomeration ${ }^{25}$. The developed morphology of our samples, is a result of unavoidable coalescence and coarsening of the clusters on the template at the deposition temperature. For example a 20 min deposition results in a fragmentary morphology of partly interconnected gold aggregates, whereas a 1 min deposition produces a dispersion of separate gold particles, see Fig. 1. Based on

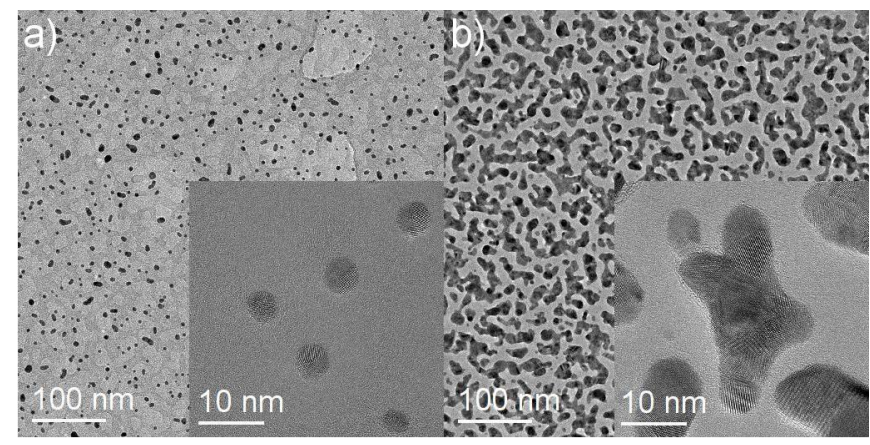

FIG. 1: HR-TEM micrographs of (a) single clusters by 1 min deposition and (b) cluster-assembled aggregates by 20 min deposition (sample C).

rigorous image analysis of HR-TEM micrographs, similar to those presented in Fig. 1, about $7.9 \pm 1.1 \%$ of the surface area of the 1 min deposited sample was covered with $\mathrm{Au}$ clusters, with an average cross-sectional area of $26 \mathrm{~nm}^{2}$. Derived from AFM measurements the corresponding cluster height is $5.1 \pm 1.8 \mathrm{~nm}$. As the cluster shape is noted to be a slightly flattened sphere, the total volume of the gold confined in the sample deposited for 1 min is estimated to be $(2.5 \pm 0.6) 10^{-8} \mathrm{~cm}^{3}$. By varying the deposition time the volume of the gold confined in the analysed samples could be adjusted, as the deposited volume is linearly dependent on deposition time. In this work three different samples, with different volumes of gold, were analysed, see Table I.

TABLE I: Summary of the samples showing the total volume of $\mathrm{Au}$ confined in them and the deposition time.

\begin{tabular}{|c|c|c|c|}
\hline sample & symbol & deposition time $(\mathrm{min})$ & volume $10^{-8}\left(\mathrm{~cm}^{3}\right)$ \\
\hline & & 1 & $2.5 \pm 0.6$ \\
\hline A & $\boldsymbol{\Delta}$ & 5 & $12.5 \pm 2.9$ \\
\hline B & $\mathbf{D}$ & 10 & $25.0 \pm 5.7$ \\
\hline C & $\bullet$ & 20 & $49.9 \pm 11.4$ \\
\hline
\end{tabular}

In Fig. 1 it can be seen that after 1 min deposition the clusters are well separated. The situation will change after 5 minutes and the probability for a significant interaction between two clusters is then higher but frequent co- 
alescence of clusters is not yet probable. As cluster dose is increased cluster coalescence will also increase. This leads to a lower surface-to-volume ratio for the samples with a higher cluster volume, with the lowest value occurring for sample C. According to AFM images there is only a 10-fold increase in surface area for sample C, the corresponding volume increase is 20 -fold for the 1 min sample. The interaction between clusters depends on remaining boundaries and thereby also affects the interacting spins.

Magnetization measurements were performed on all samples (Table I), but no magnetic signal could be detected after 1 min deposition. Fig. 2 shows magnetization of the deposited nanoparticle samples. The magnetization is seen to exhibit characteristic ferromagnetic hysteresis of the samples $\mathrm{B}$ and $\mathrm{C}$ with the remanent $\left(\mathrm{M}_{R}\right)$ and saturation $\left(\mathrm{M}_{S}\right)$ magnetizations only slightly changing with temperature (Fig 3). The coercivities $\left(\mathrm{H}_{C}\right)$ of samples $\mathrm{B}$ and $\mathrm{C}$ decrease as a function of temperature due to thermal excitations as expected. For sample A the remanent magnetization is nearly zero and the coercivity could not be clearly distinguished. The magnetization curve does not follow a clear trend as can be seen on the right hand side of Fig. 2. The magnetization of sample A exhibits a typical superparamagnetic (SPM) behaviour, i.e., typical Langevin function like behaviour (lack of coercivity and remanence and no saturation), see
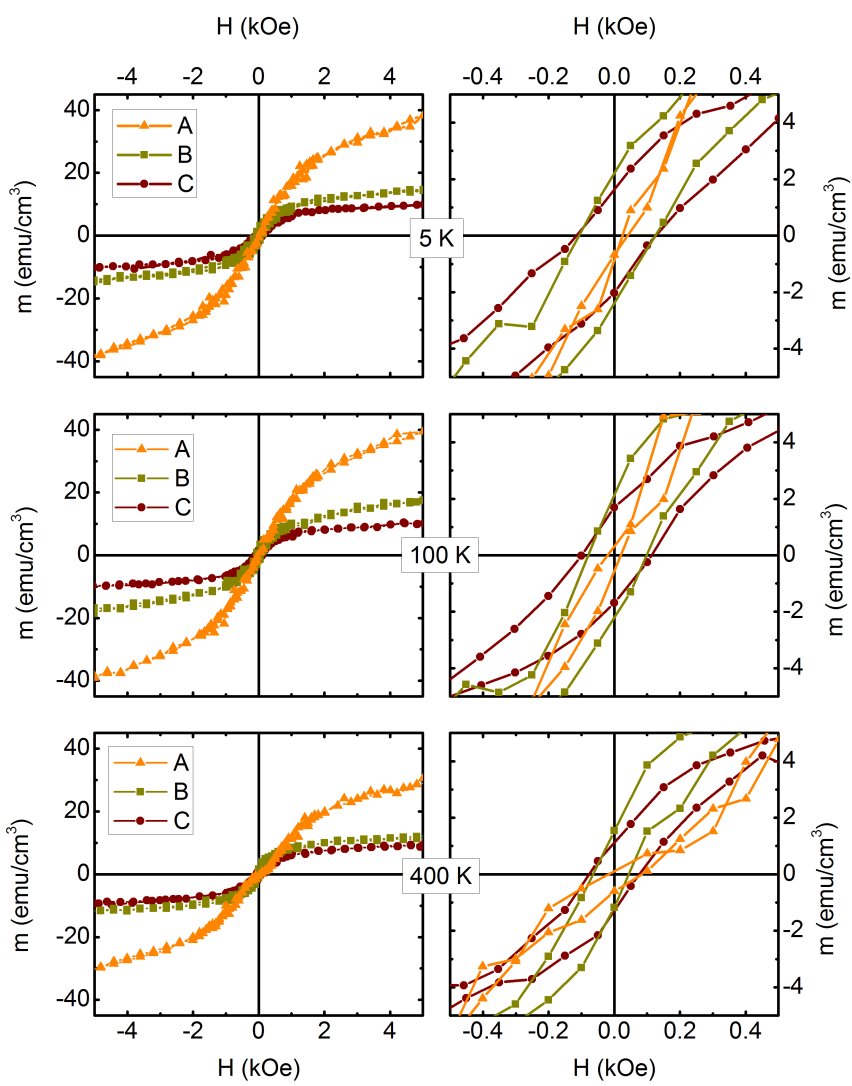

FIG. 2: Magnetization as a function of magnetic field measured at $5 \mathrm{~K}, 100 \mathrm{~K}$ and $400 \mathrm{~K}$.
Fig. 2 and supplementary information ${ }^{8}$. This SPM behaviour is not however unexpected as it is a typical behaviour of small non-interacting ferromagnetic element clusters ${ }^{26}$. The experimental uncertainty in the $\mathrm{m}(\mathrm{H})$ measurements was $\sim 10^{-2} \mathrm{emu} / \mathrm{cm}^{3}$. The error bars are within the used symbols in Fig. 2, thereby the hysteresis can unambiguously be identified at all temperatures up to $400 \mathrm{~K}$, and the expected volume dependence can be seen. From the maximum moments at saturation the saturation moments $\left(\mu_{s}\right)$ of $0.02-0.03 \mu_{B}$ /atom for sample $\mathrm{B}$ and $\sim 0.02 \mu_{B}$ /atom for sample $\mathrm{C}$ could be extracted. The corresponding highest magnetization value for sample $\mathrm{A}$ is between 0.06 and $0.08 \mu_{B}$ /atom. These values are in accordance with values provided by theoretical predictions $^{10-12}$.

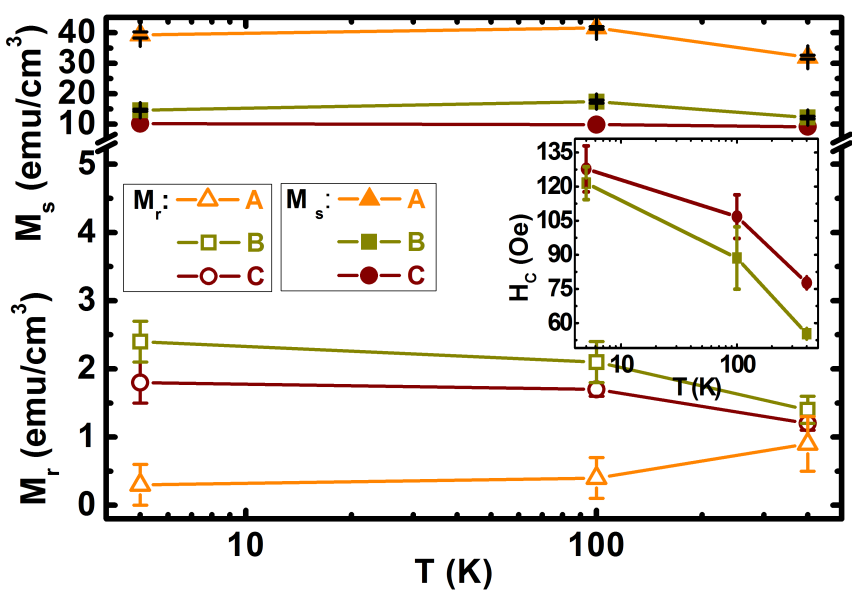

FIG. 3: Saturation, remanence and coercivity as a function of temperature.

Magnetization temperature dependence is presented in Fig. 4. After cooling to the terminal temperature of $\sim 1.8$ $\mathrm{K}$ in zero magnetic field, magnetization was measured as a function of temperature up to $\sim 400 \mathrm{~K}$ in applied magnetic fields (ZFC) of 150 and 500 Oe. After this the measurements continued while the sample was cooled back to the terminal temperature (FC). The measured magnetization is found to exhibit irreversibility between ZFC and FC branches in all applied fields, see Fig. 4. As can be seen the magnetization goes through a broad maximum spanning from cryogenic temperatures to $\sim 400 \mathrm{~K}$, after cooling in zero field. The temperature at which the curves split $\left(T_{i r r}\right)$ corresponds to the onset of the irreversibility. The FC behaviour of all samples differ from the typical SPM exponential 1/T behaviour at the measured temperature range due to high blocking temperatures and CSSG at low temperatures.

Gold clusters, in case of sample A, exhibit Langevin type of superparamagnetic core-shell behaviour up to the dilute/percolation limit. As the cluster concentration exceeds the percolation limit, magnetization decreases rapidly as the size of the coalesced clusters increases. When clusters coalescence the internal core-valence spin interaction of isolated clusters is disturbed due to dipole 

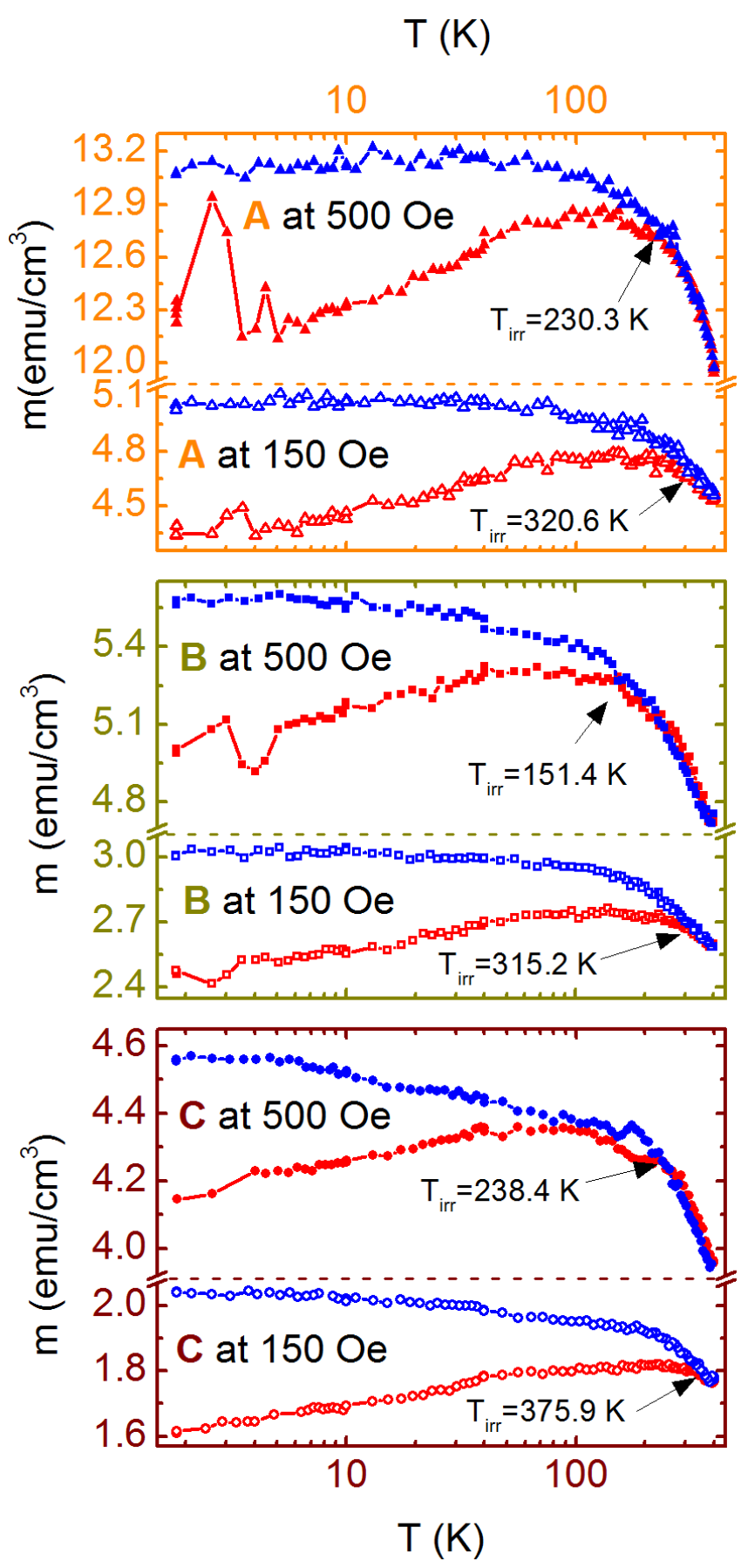

FIG. 4: Temperature dependence of magnetization in magnetic fields of 150 and 500 Oe.

and exchange interactions. Sample B presents a combination of ferromagnetic and Langevin types of interactions, as part of the clusters are connected while others still remain isolated. In fact the maximum field magnetization of samples $\mathrm{A}$ and $\mathrm{B}$ decreases at $400 \mathrm{~K}$ by about the same amount most probably due to thermal excitations of isolated super spins. Sample C, in turn, exhibits saturated ferromagnetic response as the cluster density exceeds the dilute/percolation limit. The ferromagnetism in the agglomerated magnetic clusters originates most likely from the correlated super spin glass (CSSG) and/or the ferromagnet with wandering axis (FWA) interaction mechanism.

The previous statement/cause is supported by the field cooled magnetization behaviour which shows only weak temperature dependence at low temperatures. The ZFC field response exhibits a blocking temperature with widening range as a function of cluster density (the size distribution of magnetic cluster assemblies increases/widens accordingly). A smaller low temperature peak can be distinguished for samples A and B that is due to core shell alignment / super spin alignment. The field cooled (FC) behaviour of sample A is related to SPM with high blocking temperature.

\section{CONCLUSIONS}

With all precautions taken regarding impurities and background signals we present convincing evidence of ferro- and superparamagnetism in assemblies of bare gold nanoclusters. This is demonstrated by the characteristic ferromagnetic hysteresis with the temperature dependent saturation magnetization, remanence and coercivity of Au nanoaggregates (samples B and C), as well as superparamagnetic behavior of $\mathrm{Au}$ nanoparticle aggregate dispersions (sample A). The magnetic behaviour changes from superparamagnetic to ferromagnetic due to changes in the interactions between particles. Enhanced surface-to-volume atomic ratio favours ferromagnetism in the case of bare $\mathrm{Au}$ nanoparticles and in the case of the present gold cluster agglomerates the behaviour is analogous to that of transition metal clusters ${ }^{18}$.

\section{ACKNOWLEDGEMENTS}

The doctoral program in Materials Research and Nanosciences (MATRENA) of Helsinki University is acknowledged for financial support. This research made use of the OtaNano - Low Temperature Laboratory infrastructure of Aalto University.

\section{CONFLICT OF INTEREST}

The authors declare that there is no conflict of interest regarding the publication of this article.

\section{REFERENCES}

${ }^{1}$ G. L. Nealon, B. Donnio, R. Greget, J.-P. Kappler, E. Terazzi, and J.-L. Gallani, "Magnetism in gold nanoparticles." Nanoscale 4, 5244-5258 (2012). 
${ }^{2}$ R. Sato, S. Ishikawa, H. Sato, and T. Sato, "Magnetic order of Au nanoparticle with clean surface," J. Magn. Magn. Mater. 393, 209-212 (2015).

${ }^{3}$ S. Ishikawa and T. Sato, "Verification of Ferromagnetism in Au Nanoparticles with Clean Surface," in Proc. 12th Asia Pacific Phys. Conf. (Journal of the Physical Society of Japan, 2014).

${ }^{4}$ C.-Y. Li, C.-M. Wu, S. K. Karna, C.-W. Wang, D. Hsu, C.J. Wang, and W.-H. Li, "Intrinsic magnetic moments of gold nanoparticles," Phys. Rev. B 83, 174446 (2011).

${ }^{5}$ C.-M. Wu, C.-Y. Li, Y.-T. Kuo, C.-W. Wang, S.-Y. Wu, and W.-H. Li, "Quantum spins in Mackay icosahedral gold nanoparticles," J. Nanoparticle Res. 12, 177-185 (2010).

${ }^{6}$ H. Hori, T. Teranishi, Y. Nakae, Y. Seino, M. Miyake, and S. Yamada, "Anomalous magnetic polarization effect of Pd and Au nano-particles," Phys. Lett. A 263, 406-410 (1999).

${ }^{7}$ H. Hori, Y. Yamamoto, T. Iwamoto, T. Miura, T. Teranishi, and M. Miyake, "Diameter dependence of ferromagnetic spin moment in Au nanocrystals," Phys. Rev. B 69, 174411 (2004).

${ }^{8}$ Y. Yamamoto, T. Miura, M. Suzuki, N. Kawamura, H. Miyagawa, T. Nakamura, K. Kobayashi, T. Teranishi, and H. Hori, "Direct Observation of Ferromagnetic Spin Polarization in Gold Nanoparticles," Phys. Rev. Lett. 93, 116801 (2004).

${ }^{9}$ H. Yamamoto, Y.; Hori, "Direct Observation of the ferromagnetic spin polarization in gold nanoparticles: A Review," Rev.Adv.Mater.Sci. 12, 23-32 (2006).

${ }^{10}$ W. Luo, S. J. Pennycook, and S. T. Pantelides, "s-Electron ferromagnetism in gold and silver nanoclusters." Nano Lett. 7, 3134-7 (2007).

${ }^{11}$ R. Magyar, V. Mujica, M. Marquez, and C. Gonzalez, "Densityfunctional study of magnetism in bare Au nanoclusters: Evidence of permanent size-dependent spin polarization without geometry relaxation," Phys. Rev. B 75, 144421 (2007).

${ }^{12}$ S. S. Pundlik, K. Kalyanaraman, and U. V. Waghmare, "FirstPrinciples Investigation of the Atomic and Electronic Structure and Magnetic Moments in Gold Nanoclusters," J. Phys. Chem. C 115, 3809-3820 (2011).

${ }^{13} \mathrm{~K}$. Okazaki and Y. Teraoka, "Magnetic structures in metallic thin films," Surf. Sci. 433-435, 672-675 (1999).

${ }^{14}$ R. A. Guirado-López, J. Dorantes-Dávila, and G. M. Pastor, "Orbital Magnetism in Transition-Metal Clusters: From Hund's
Rules to Bulk Quenching," Phys. Rev. Lett. 90, 226402 (2003).

${ }^{15}$ F. Michael, C. Gonzalez, V. Mujica, M. Marquez, and M. Ratner, "Size dependence of ferromagnetism in gold nanoparticles: Mean field results," Phys. Rev. B 76, 224409 (2007).

${ }^{16}$ K. Koga, T. Ikeshoji, and K.-i. Sugawara, "Size- and Temperature-Dependent Structural Transitions in Gold Nanoparticles," Phys. Rev. Lett. 92, 115507 (2004).

${ }^{17}$ V. Tuboltsev, A. Savin, A. Pirojenko, and J. Räisänen, "Magnetism in nanocrystalline gold." ACS Nano 7, 6691-6699 (2013).

${ }^{18} \mathrm{~J}$. BANSMANN, S. BAKER, C. BINNS, J. BLACKMAN, J. BUCHER, J. DORANTESDAVILA, V. DUPUIS, L. FAVRE, D. KECHRAKOS, and A. KLEIBERT, "Magnetic and structural properties of isolated and assembled clusters," Surf. Sci. Rep. 56, 189-275 (2005).

${ }^{19}$ J. F. Löffler, H.-B. Braun, and W. Wagner, "Magnetic Correlations in Nanostructured Ferromagnets," Phys. Rev. Lett. 85, 1990-1993 (2000).

${ }^{20}$ E. M. Chudnovsky, W. M. Saslow, and R. A. Serota, "Ordering in ferromagnets with random anisotropy," Phys. Rev. B 33, 251261 (1986).

${ }^{21}$ E. M. Chudnovsky, "Magnetic properties of amorphous ferromagnets (invited)," J. Appl. Phys. 64, 5770-5775 (1988).

${ }^{22}$ E. M. CHUDNOVSKY, "RANDOM ANISOTROPY IN AMORPHOUS ALLOYS," in Magn. Amorph. Met. Alloy. (WORLD SCIENTIFIC, 1995) pp. 143-174.

${ }^{23}$ I. Yamada and N. Toyoda, "Recent advances in R\&D of gas cluster ion beam processes and equipment," Nucl. Instruments Methods Phys. Res. Sect. B Beam Interact. with Mater. Atoms 241, 589-593 (2005).

${ }^{24}$ J. B. Anderson and J. B. Fenn, "Velocity Distributions in Molecular Beams from Nozzle Sources," Phys. Fluids 8, 780 (1965).

${ }^{25}$ C. Binns, "Nanoclusters deposited on surfaces," Surf. Sci. Rep. 44, 1-49 (2001).

${ }^{26} \mathrm{~V}$. Singh and V. Banerjee, "Ferromagnetism, hysteresis and enhanced heat dissipation in assemblies of superparamagnetic nanoparticles," J. Appl. Phys. 112, 114912 (2012). 